\title{
High Performance CMOS Level up Conversion for Systems with Low-Voltage Power Supply
}

\author{
José-Carlos García and Juan A. Montiel-Nelson \\ Institute for Applied Microelectronics. University of Las \\ Palmas de Gran Canaria, Spain \\ \{jcgarcia, montiel\}@iuma.ulpgc.es
}

\author{
S. Nooshabadi \\ Department of Electrical and Computer Engineering, \\ Michigan Technological UniversityMichigan, USA \\ saeid@mtu.edu
}

\begin{abstract}
A single supply CMOS non-inverting level shifter circuit, $c c m-l s$, for converting input signals from $0.3 \mathrm{~V}$ up to $0.7 \mathrm{~V}$ is presented. The circuit is optimized and pre-layout simulated in a 65nm CMOS process technology. At the target design voltage of $0.3 \mathrm{~V}$, the level shifter has a propagation delay of $1.06 \mathrm{~ns}$, an energy consumption of only $0.79 \mathrm{pJ}$, and energy-delay product of $0.84 \mathrm{pJns}$ for capacitive load of $1 \mathrm{pf}$. Simulation results are compared to other similar published works at a frequency of $\mathbf{5 0 0}$ MHz, and it shown that the proposed circuit outperforms them.
\end{abstract}

Keywords: Up converter; voltage domain; low-energy; lowvoltage; single supply; level shifting; bootstrap; CMOS design

\section{INTRODUCTION}

In recent years, there has been considerable research effort in the development of low-energy consumption level shifters, with very small supply voltage and improved subthreshold range due mainly to their low-power application in portable systems. Several level converters topologies have been proposed to achieve voltages in the order of a few $\mathrm{mV}$ with a low-voltage power supply. Multi-threshold voltage CMOS processes are used in the design of voltage level shifter in order to reduce delay (with low-threshold transistor) and power (with high threshold transistor). The design presented in [1] uses a level shifter to connect the output of a $2: 1$ multiplexer operating at supply voltage of $0.2 \mathrm{~V}$ to a static random access memory (SRAM) operating at $1 \mathrm{~V}$ supply voltage; the circuit was implemented in a 90nm CMOS technology. The work in [2], reported a level shifter using a diode-connected transistor and dynamic current switching. Requiring a control circuit, only during the high-to-low transitions of the output, a current is forced into the diode-connected device. The proposed circuit was fabricated in both $40 \mathrm{~nm}$ and $180 \mathrm{~nm}$ standard CMOS technologies. The level converter in [3] achieves low power by employing a new topology based on a reduced-swing buffer while a pass transistor enhances the speed of the fall transition so that the conversion delay can be significantly improved. Measurement results from a test chip fabricated in $65 \mathrm{~nm}$ CMOS technology show low leakage and high speed. The reported level shifter can convert deep sub-threshold voltage as low as $100 \mathrm{mV}$ to the super-threshold voltage of $1.2 \mathrm{~V}$.

This paper deals with a high performance level up shifter, $\mathrm{ccm}$-ls, designed to convert near-threshold voltages to power supply voltage level. The remainder of this work is organized as follows. Section II presents the proposed voltage level up shifter. In Section III, pre-layout simulation results are discussed and evaluated. Section IV is dedicated to compare the proposed design with other reported circuit, $s s-l s$, modified [4]. Section V describes the comparison between $\mathrm{ccm}$-ls and other CMOS level converters of the state-of-the-art. Postlayout simulation details are presented in Section VI. Finally, Section VII concludes the work.

\section{CIRCUIT TOPOLOGIES AND OPERATION}

Fig. 1 shows a level shifter termed $s s-l s$ based on a bootstrap capacitor (MPC) which is charged to supply voltage (Vddh), this enables to increase the output level of node 3 during low to high transitions at node 1. The drain of MP6 charges MPC in conjunction with MP2 up to Vddh-Vddl. The current source (MP5 and MP6) is only turned on during a low to high transition at the input node (in). When MN2 is turned on, MPC provides a negative voltage that turns MN3 off quickly and reduces the static leakage current.

In this work we propose a 6-stage circuit shown in Fig. 2, termed $\mathrm{ccm}$-ls. The first inverter has a diode-connected transistor (MN7) for reducing the voltage at node 6 to Vddh$0.3 \mathrm{~V}$, and for minimizing the static power consumption when node in is in high logic level, where MN1 is on.

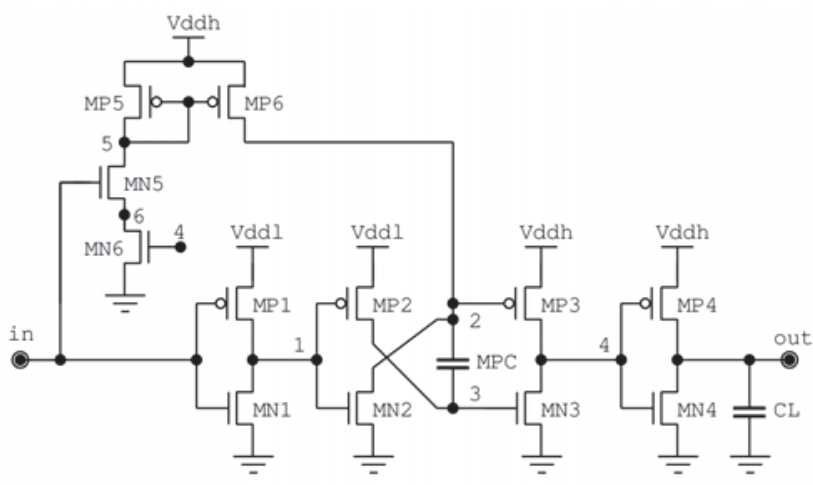

Figure 1. The circuit diagram for $s s-l s$, modified [4] 


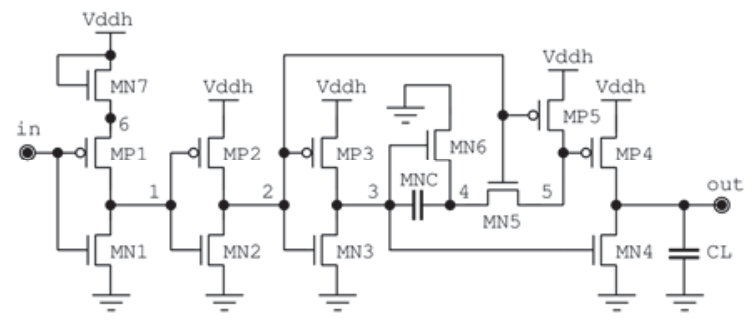

Figure 2. Schematic for $c c m$ - $l s$ level up shifter.

The principal stage is the fourth, which consists of MN6 and MNC. If node in is low, node 3 is high, and MNC is charged through MN6, MN4 is also turned on and the output, node out, is set to low. In addition, in that case, node 2 is low, MP3, and MP5 conduct, whereas MN5 and MP4 are turned off. If a high logic level is applied at node in, node 1 and 3 are low, MN5 conducts, and MP4 quickly turns on, so that output is pulled up. Table I shows channel widths for transistors in both circuits (length $=65 \mathrm{~nm}$ ). Note that active area for $s s-l s$ is three times higher than that for $c c m-l s$. Sizing for both circuits is obtained for the best figure-of-merit criterion of energy-delayproduct (EDP). In contrast to $s s-l s, c c m-l s$ is capable of operating correctly with an input signal of $0.3 \mathrm{~V}$ for a supply voltage of $0.7 \mathrm{~V}$, and a capacitive load of $1 \mathrm{pF}$, with a propagation time delay of $1.06 \mathrm{~ns}$, the energy consumption of $0.79 \mathrm{pJ}$, and EDP of $0.84 \mathrm{pJns}$.

\section{Simulation RESUltS}

Fig. 3 illustrates simulation waveforms of both designs when the input signal has a voltage level of $0.38 \mathrm{~V}$, Vddl is $0.38 \mathrm{~V}$, and $\mathrm{Vddh}$ is $0.7 \mathrm{~V}$. Operating frequency is $500 \mathrm{MHz}$, and the load capacitance is $600 \mathrm{fF}$. Fig. 3 shows that propagation time delay for $s s-l s$ is $1.35 \mathrm{~ns}$ and for $c c m-l s$ is $0.89 \mathrm{~ns}$. Each circuit is simulated adding a parasitic capacitor of $25 \mathrm{fF}$ and an inverter with $80 \mu \mathrm{m} / 20 \mu \mathrm{m}$ PMOS/NMOS channel widths ratio connected to node out. It is observed that they both exhibit a rail-to-rail switching characteristic.

Fig. 4 presents optimized EDP when the input signal is $0.38 \mathrm{~V}$ and the capacitive load ranges from 60 to $600 \mathrm{fF}$ for both

TABLE I. Sizing For PMOS AND NMOS TRANSISTORS

\begin{tabular}{|c|c|c|c|c|c|}
\hline \multicolumn{3}{|c|}{ Ss-ls $\left(\right.$ Active area $\left.=103.35 \mu \mathrm{m}^{2}\right)$} & \multicolumn{3}{|c|}{ Ccm-ls $\left(\right.$ Active area $=30.43 \mu \mathrm{m}^{2}$ ) } \\
\hline Transistor(s) & Type & Width $(\mu \mathrm{m})$ & Transistor(s) & Type & Width $(\mu m)$ \\
\hline MP1 & $\mathrm{P}$ & $28 \times 10$ & MP1 & $\mathrm{P}$ & $6 \times 10$ \\
\hline MP2 & $\mathrm{P}$ & $4 \times 10$ & MP2 & $\mathrm{P}$ & $0.4 \times 10$ \\
\hline MP3 & $\mathrm{P}$ & $15 \times 10$ & MP3, MP4 & $\mathrm{P}$ & $2 \times 10$ \\
\hline MP4 & $\mathrm{P}$ & $6 \times 10$ & MP5 & $\mathrm{P}$ & $1 \times 10$ \\
\hline MP5, MP6 & $\mathrm{P}$ & $2 \times 10$ & $\mathrm{MNC}$ & $\mathrm{N}$ & $0.025 \times 10$ \\
\hline MPC & $\mathrm{P}$ & $50 \times 10$ & MN1, MN7 & $\mathrm{N}$ & $10 \times 10$ \\
\hline MN1 & $\mathrm{N}$ & $5 \times 10$ & MN2 & $\mathrm{N}$ & $2 \times 10$ \\
\hline MN2 & $\mathrm{N}$ & $12 \times 10$ & MN3, MN5 & $\mathrm{N}$ & $0.2 \times 10$ \\
\hline $\mathrm{MN} 3$ & $\mathrm{~N}$ & $4 \times 10$ & $\mathrm{MN} 4$ & $\mathrm{~N}$ & $8 \times 10$ \\
\hline MN4 & $\mathrm{N}$ & $1 \times 10$ & MN6 & $\mathrm{N}$ & $5 \times 10$ \\
\hline MN5, MN6 & $\mathrm{N}$ & $15 \times 10$ & - & - & - \\
\hline
\end{tabular}

$\mathrm{VThn}=0.28 \mathrm{~V}$ and VThp $=-0.2 \mathrm{~V}$. The channel length for all transistors is $65 \mathrm{~nm}$.

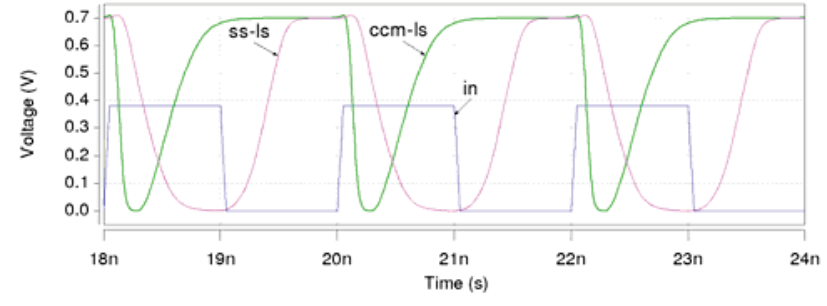

Figure 3. Waveforms using a capacitive load of $600 \mathrm{fF}$.

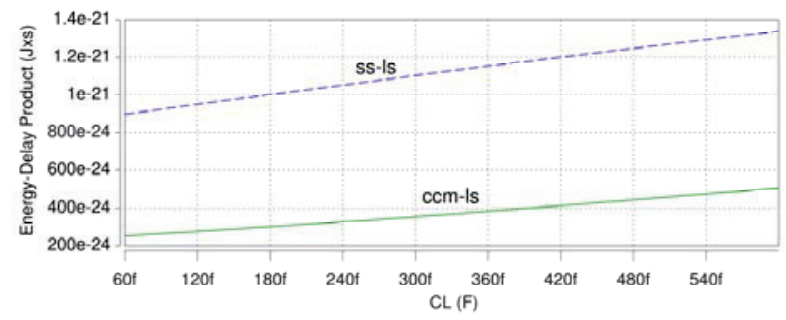

Figure 4. Energy-delay-product versus capacitive load for $s s-l s$ and $c c m-l s$ circuits.

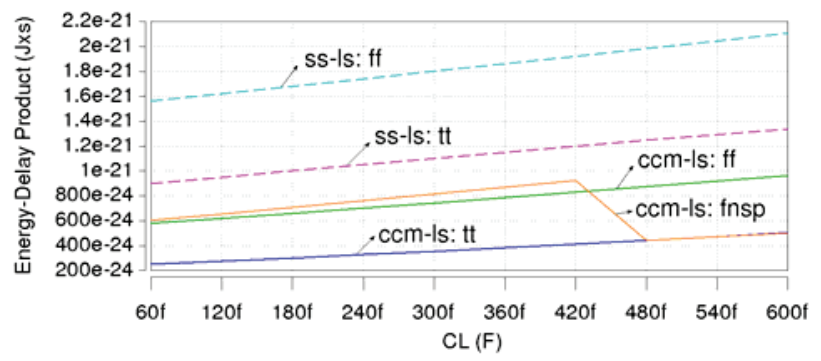

Figure 5. Energy-delay-product for $s s-l s$ and $c c m$ - $l s$ circuits at different process corners.

$s s-l s$ and $c c m-l s$ circuits. Variation in load capacitance effects on the $s s-l s$ is far more than on $c c m-l s$.

Fig. 5 compares EDP results for typical-typical and fastfast process corners considering a working frequency of $500 \mathrm{MHz}$. For fast NMOS-slow PMOS case, $s s-l s$ fails to operate correctly, unless the input frequency is reduced to $320 \mathrm{MHz}$. For the slow-slow process corner the, frequency of operation needs to reduce to below $160 \mathrm{MHz}$ for the correct operation of both $s s-l s$ and $c c m-l s$. For the process corner of slow NMOS-fast PMOS, ccm-ls works correctly at $400 \mathrm{MHz}$. In contrast $s s$ - $l s$ can only achieve that a maximum frequency of $250 \mathrm{MHz}$.

A decrease of a $10 \%$ in $\mathrm{Vddh}$ reduces the operational frequency to $200 \mathrm{MHz}$ for the case of $c c m-l s$. For $s s-l s$ the corresponding reduction in frequency is $400 \mathrm{MHz}$. However, both crcuits perform correctly when Vddh is increased by $10 \%$. In this case $c c m-l s$ presents a $60 \%$ lower EDP than $s s-l s$ at $500 \mathrm{MHz}$. EDP comparisons at $200 \mathrm{MHz}$, across a range of capacitive loading, are shown in Fig. 6 for the Vddh variation of $\pm 10 \%$ at $0.7 \mathrm{~V}$. Ccm-ls exhibits a much better EDP. 


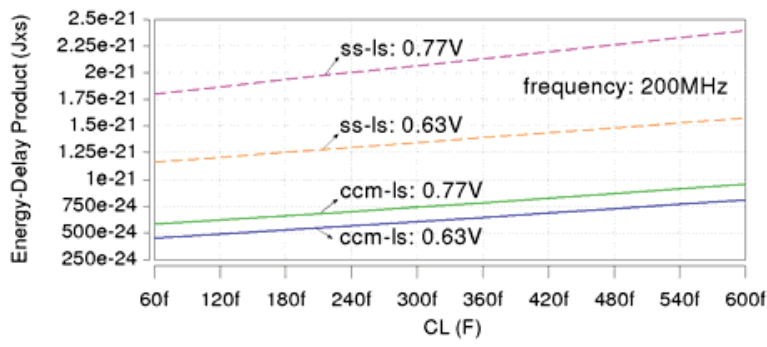

Figure 6. EDP for $s s-l s$ and $c c m$ - $l s$ circuits evaluated for different voltage corners at $200 \mathrm{Mhz}$ when the input signal level is $0.38 \mathrm{~V}$.

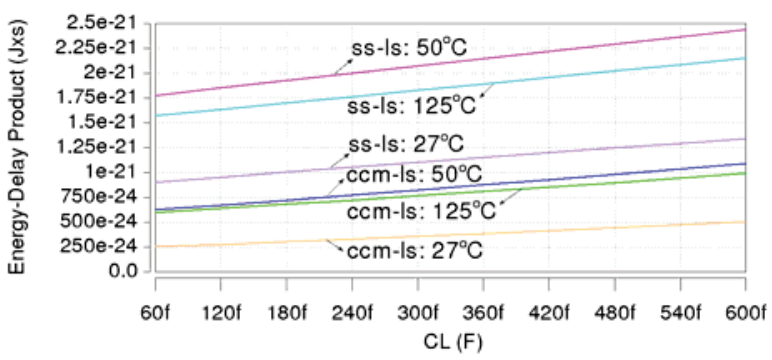

Figure 7. EDP for $s s-l s$ and $c c m-l s$ circuits evaluated for different positive temperatures at $500 \mathrm{MHz}$ when the input signal level is $0.38 \mathrm{~V}$.

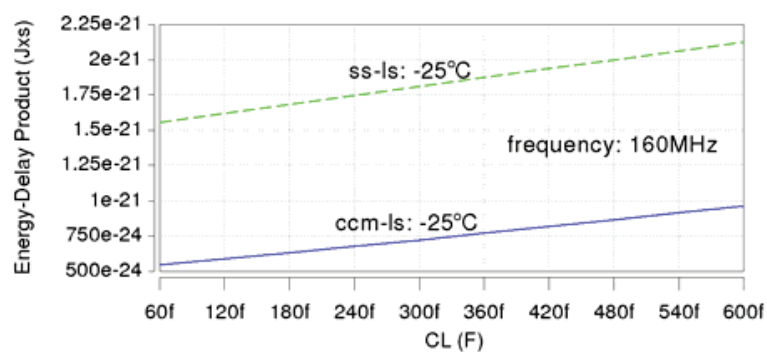

Figure 8. EDP for $s s-l s$ and $c c m-l s$ circuits evaluated for a negative temperatures at $160 \mathrm{MHz}$ when the input signal level is $0.38 \mathrm{~V}$.

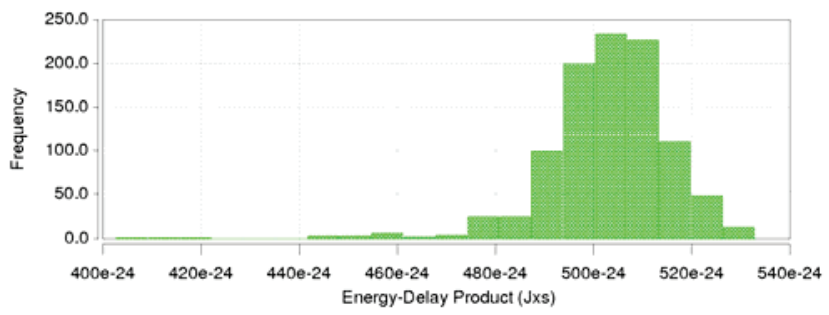

Figure 9. Histogram for EDP of $c c m-l s$ at $500 \mathrm{MHz}$ when the input signal level is $0.38 \mathrm{~V}$

Simulations results for three different positive workig temperatures are shown in Fig. 7. Ccm-ls exhibits the best $\mathrm{EDP}$ at $27^{\circ} \mathrm{C}$. For the negative working temperature of $-25^{\circ} \mathrm{C}$,

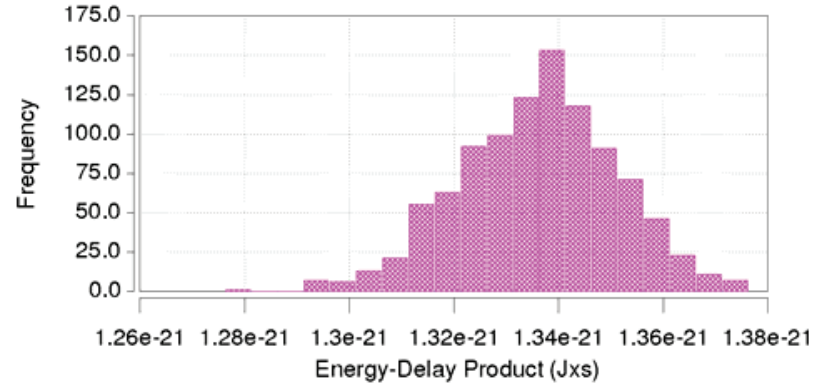

Figure 10. Histogram for EDP of $s s-l s$ at $500 \mathrm{Mhz}$ when the input signal level is $0.38 \mathrm{~V}$.

the input signal frequency must be decreased to $160 \mathrm{MHz}$ for both $c c m$ - $l s$ and $s s-l s$. Fig. 8 shows the EDP comparison for the case in which the temperature is $-25^{\circ} \mathrm{C}$. At $600 \mathrm{fF}$ output loading condition EDP for $\mathrm{ccm}-\mathrm{ls}$ is $54.7 \%$ lower than the required for $s s-l s$. To analyze how mismatch in the device paramters affects the performance of $\mathrm{ccm}$ - $\mathrm{s}$, a 1000-point Monte Carlo simulation is shown in Fig. 9. The mean for EDP, $\mu$, is 502.89pJps and standard deviation, $\sigma$, is $13.01 \mathrm{pJps}$, and normalized variance value of $\sigma / \mu$ of 0.02 . The EDP shows a very small sensitivity toward device parameters mismatch. More than a $70 \%$ of all simulated results for $\mathrm{ccm}-\mathrm{ls}$ fall between 490 and 520pJps. Fig. 10 exposes the similar histogram for $s s-l s$. The spread for $s s-l s$ has a mean of $1336 \mathrm{pJps}$ and a standard deviation of $14.97 \mathrm{pJps}$. The normalized variance value, $\sigma / \mu$, is 0.01 , lower than that required for $c \mathrm{~cm}-\mathrm{ls}$.

\section{PRE-LAYOUT COMPARISON RESULTS}

The two circuits are simulated in 65nm CMOS technology process with input supply voltage of $0.38 \mathrm{~V}$. From the results, it is observed that propagation time delay and EDP for $\mathrm{ccm}$ - $\mathrm{ls}$ are $34 \%$ and $62.4 \%$ lower than those for $s s-l s$, respectively. Considering only the power supply of Vddh, with the load of $\mathrm{CL}=600 \mathrm{fF}$, the static currents for $c \mathrm{~cm}$-ls for the cases of low and a high input levels are $0.13 \mu \mathrm{A}$ and $0.28 \mu$, respectively. In other words, $c c m-l s$ has got $72 \%$ and $54 \%$ lower static current than $s s-l s$, respectively. Note that $s s-l s$ uses a second power supply, Vddl that introduces an additional static power consumption.

\section{COMPARISON WITH OTHER PUBLISHED LEVEL SHIFTERS}

Table II presents the differences between $\mathrm{ccm}$ - $\mathrm{ls}$ and references [1]-[4]. Our converter denotes pre-layout results as [1] but $\mathrm{ccm}$-ls uses a higher capacitive load. Delay for [1] is roughly ten times higher than obtained for $\mathrm{ccm}-\mathrm{ls}$. However, [1] has lower active area and power consumption than $\mathrm{ccm}-\mathrm{ls}$ apart from a wider input voltage range of conversion. In [2] is used a CMOS technology with the lowest gate length than the rest of designs in Table II. In addition, [2] improves the area, power consumption and conversion range in comparison with ccm-ls but requires a higher EDP. Circuit in [3] uses the same 
TABLE II. COMPARISON TO THE STATE-OF-THE-ART

\begin{tabular}{|c|c|c|c|c|c|}
\hline Design & [1] & [2] & [3] & [4] & ccm-ls \\
\hline Year & 2016 & 2018 & 2018 & 2017 & 2018 \\
\hline Technology & 90nm CMOS & 40nm CMOS & $65 \mathrm{~nm}$ CMOS & 180nm CMOS & $65 \mathrm{~nm}$ CMOS \\
\hline Results & Pre-layout & Measure & Measure & Measure & Pre-layout \\
\hline Area & $1.12 \mu \mathrm{m}^{2}$ & $12 \mu \mathrm{m}^{2}$ & $7.45 \mu \mathrm{m}^{2}$ & $229.5 \mu \mathrm{m}^{2}$ & $30.43 \mu \mathrm{m}^{2}$ \\
\hline Delay & 10.99ns@0.2V & 80ns@0.3V & 7.5ns@0.3V & 29ns@0.4V & $1.06 \mathrm{~ns}$ \\
\hline Power & $1.52 \mathrm{nW}$ & 0.18nW@0.35V & $123.8 \mathrm{nW}$ & $61.5 \mathrm{nW}$ & $395 \mu \mathrm{W}$ \\
\hline Frequency & Not available & $10 \mathrm{KHz}$ & $1 \mathrm{MHz}$ & $500 \mathrm{KHz}$ & $500 \mathrm{MHz}$ \\
\hline EDP (FoM) & Not available & $1.44 \mathrm{pJns}$ & $0.92 \mathrm{pJns}$ & $3.56 \mathrm{pJns}$ & $0.84 \mathrm{pJns}$ \\
\hline Load & $1 \mathrm{fF}$ & 4 inverters & Not available & $10 \mathrm{fF}$ & $1 \mathrm{pF}$ \\
\hline Voltages (V) & 0.1 to 1 & 0.05 to 1.1 & 0.1 to 1.2 & 0.33 to 1.8 & 0.3 to 0.7 \\
\hline
\end{tabular}

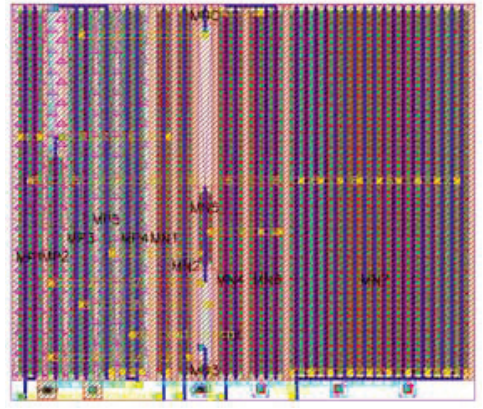

Figure 11. Layout for $c c m$ - $l s$ with the core area of $\mathrm{W} \times \mathrm{H}$ is $12.63 \mu \mathrm{m} \times$ $10.85 \mu \mathrm{m}$.

power consumption and the input voltage range but its output capacitive load is unknown. Delay for [3] is 7 times higher than ccm-ls. On the other hand, design in [4] presents a chip area, delay, EDP, and conversion range greater than required for $\mathrm{ccm}$-ls using a capacitive load that is 100 times smaller.

\section{Post-layout Simulation Results}

It is necessary to reduce the operating frequency from $500 \mathrm{MHz}$ to $400 \mathrm{MHz}$ when the input signal reaches the limit of $0.7 \mathrm{~V}$ and the output is set to $0.7 \mathrm{~V}$ with a CL of $50 \mathrm{fF}$. We use layout shown in Fig. 11 to evaluate the effective input conversion range of $\mathrm{ccm}$-ls at $400 \mathrm{MHz}$ operating frequency. It is demostrated that it is feasible to work with the input signal range of 0.38 to $0.7 \mathrm{~V}$, with the output voltaje of $0.7 \mathrm{~V}$ while achieving a low EDP. Table III prsenents the the transistor sizing for the layout in Fig. 11 using optimized EDP strategy and output full swing at $50 \mathrm{fF}$ load capacitance. In this case, EDP is $0.46 \mathrm{pJns}$, and delay is $1.24 \mathrm{~ns}$ at $400 \mathrm{MHz}$.

We can maintain an input voltage in the range of $0.3 \mathrm{~V}$ to $0.7 \mathrm{~V}$ at an increased operating frequency up to $500 \mathrm{MHz}$ at $\mathrm{CL}$ to $525 \mathrm{fF}$ with a design resizing. For this purpose is necessary to modify the width for MN1 $(10 \times 10 \mu \mathrm{m}), \mathrm{MN} 2$ $(2 \times 10 \mu \mathrm{m}), \mathrm{MN} 3(0.1 \times 10 \mu \mathrm{m})$, and MP1 $(6 \times 10 \mu \mathrm{m})$. Active area for the resized design is $30.50 \mu \mathrm{m}^{2}$. The core area for $c c m-l s$ is $16.35 \mu \mathrm{m} \times 10.85 \mu \mathrm{m}(\mathrm{WxH})$. The EDP for this layout is $0.65 \mathrm{pJns}$ and the delay is $1.47 \mathrm{~ns}$.

TABLE III. POST-LAYOUT SIZING FOR CCM-LS OPERATING AT 400MHZ

\begin{tabular}{||c|c|c||}
\hline \multicolumn{3}{|c||}{ Ccm-ls $\left(\right.$ Active area $\left.=21.35 \mu \mathrm{m}^{2}\right)$} \\
\hline \hline Transistor(s) & Type & Width $(\boldsymbol{\mu m})$ \\
\hline MP1, MP3 and MP4 & $\mathrm{P}$ & $2 \times 10$ \\
\hline MP2 & $\mathrm{P}$ & $0.6 \times 10$ \\
\hline MP5 & $\mathrm{P}$ & $1 \times 10$ \\
\hline MNC and MN3 & $\mathrm{N}$ & $0.025 \times 10$ \\
\hline MN1 and MN2 & $\mathrm{N}$ & $1 \times 10$ \\
\hline MN4 & $\mathrm{N}$ & $2 \times 10$ \\
\hline MN5 & $\mathrm{N}$ & $0.2 \times 10$ \\
\hline MN6 & $\mathrm{N}$ & $3 \times 10$ \\
\hline MN7 & $\mathrm{N}$ & $18 \times 10$ \\
\hline
\end{tabular}

\section{CONCLUSIONS}

A low power bootstrapped CMOS level shifter is designed in order to reduce power consumption and enhance the speed of switch for up shifting and driving a large load. According to the HSPICE simulation results, the proposed level converter reduces the EDP figure-of-merit by $62.4 \%$ compared to a similar reported design at $500 \mathrm{MHz}$ and with a capacitive load of $600 \mathrm{fF}$.

\section{ACKNOWLEDGMENT}

This work was funded by project SURF (TEC201460527C21R) of the Spanish Ministry of Economy and Competitiveness.

\section{REFERENCES}

[1] P. Gosatwar and U. Ghodeswar, "Design of voltage level shifter for multi-supply voltage design," in Proc. ICCSP, Apr. 2016, pp. 853857.

[2] R. Lotfi, M. Saberi, S. R. Hossini, A. R. Ahmadi-Mehr, and R. B. Staszewski, "Energy-efficient wide-range voltage level shifters reaching 4.2fJ/transition," IEEE Solid-State Circuits Letters, vol. 1, no. 2, pp. 34-37, Feb. 2018.

[3] V. L. Le and T. T.-H. Kim, "An area and energy efficient ultra-low voltage level shifter with pass transistor and reduced-swing output 
buffer in 65-nm CMOS," IEEE Trans. Circuits Syst. II, Exp. Briefs, vol. 65, no. 5, pp. 607-611, May 2018.

[4] E. Maghsoudloo, M. Rezaei, M. Sawan, and B. Gosselin, "A highspeed and ultra low-power subthreshold signal level shifter," IEEE Trans. Circuits Syst. I, vol. 64, no. 5, pp. 1164-1172, May 2017. 